\title{
Electromagnetic trapping of chiral molecules: orientational effects of the irradiating beam
}

\author{
David S. Bradshaw and David L. Andrews* \\ School of Chemistry, University of East Anglia, Norwich NR4 7TJ, UK \\ ${ }^{*}$ Corresponding author: d.I.andrews@uea.ac.uk
}

Received 17 December 2014; revised 19 February 2015; accepted 20 February 2015; posted 20 February 2015 (Doc. ID 231037 ); published 10 April 2015

\begin{abstract}
The photonic interaction generally responsible for the electromagnetic trapping of molecules is forward-Rayleigh scattering, a process that is mediated by transition electric dipoles connecting the ground electronic state and virtual excited states. Higher order electric and magnetic multipole contributions to the scattering amplitude are usually negligible. However, on consideration of chiral discrimination effects (in which an input light of left-handed circular polarization can present different observables compared to right-handed polarization, or molecules of opposite enantiomeric form respond differently to a set circular polarization), the mechanism must be extended to specifically accommodate transition magnetic dipoles. Moreover, it is important to account for the fact that chiral molecules are necessarily nonspherical, so that their interactions with a laser beam will have an orientational dependence. Using quantum electrodynamics, this article quantifies the extent of the energetic discrimination that arises when chiral molecules are optically trapped, placing particular emphasis on the orientational effects of the trapping beam. An in-depth description of the intricate ensemble-weighted method used to incorporate the latter is presented. It is thus shown that, when a mixture of molecular enantiomers is irradiated by a continuous beam of circularly polarized light, a difference arises in the relative rates of migration of each enantiomer in and out of the most intense regions of the beam. As a consequence, optical trapping can be used as a means of achieving enantiomer separation. (c) 2015 Optical Society of America
\end{abstract}

OCIS codes: (020.7010) Laser trapping; (160.1585) Chiral media; (260.2110) Electromagnetic optics; (270.5580) Quantum electrodynamics; (290.2558) Forward scattering.

\section{INTRODUCTION}

Electromagnetic (or optical) trapping is a well-established technique used to manipulate dielectric microparticles through the application of a nonabsorbing, off-resonant laser beam. Currently, there is much interest in the application of such a gradient trapping force to solid chiral microparticles, for example, in connection with the observation of the internal helical structure of such particles $[\underline{1}, 2]$, the production of an omnidirectional chiral mirror to enable optomechanical effects [3] , and the selective manipulation of chiral objects dependent on photon helicity [4]. This paper, in contrast, deals with the optical trapping of chiral molecules or Rayleigh particles at the nanoscale, which requires a consideration of individual photonmatter interactions in the form of forward-Rayleigh scattering [5-8], namely, the concerted annihilation and creation of photons with identical energy and wave-vector. The molecular property, by which this effect is commonly mediated, is the dynamic electronic polarizability, a tensor which is cast in terms of transition electric dipole moments. Since every molecule has a nonzero electric polarizability, and any contributions associated with higher order transition moments rapidly diminish with increasing order, it is usually considered unnecessary to entertain the possible role of transition magnetic dipoles for the molecule in such electromagnetic trapping studies. However, despite a coupling strength that is usually much smaller in magnitude than its electric equivalent, considerations of magnetic dipole involvement prove to be vital in the examination of chiral discrimination effects, such as those in which left-handed circularly polarized light may produce an observable that differs from its equivalent with right-handed light. The reason is that electric and magnetic effects have fundamentally different symmetry properties with regard to spatial inversion (and time reversal).

In detail, whereas the leading contribution to forwardRayleigh scattering relates to mediation of the two lightmatter interactions (photon annihilation and photon creation) by electric dipole moments, scattering may also occur if one of these electric dipole interactions is replaced by a magnetic dipole; it is this situation that affords the largest and most significant contribution to chiral discrimination. As will be shown, this feature can be exploited in the electromagnetic 
trapping of chiral molecules. The fundamental physical observable (whether or not chiral molecules are involved) is the potential energy, since the initial and final system states are identical [9], as follows from the fact that the irradiating nonabsorbing beam emerges from the forward scattering process unchanged. This contrasts, for example, with the optically resonant process of circular dichroism - the differential absorption of left- and right-handed circularly polarized lightwhose observable is signified by a rate (as determined from the Fermi Rule) and is thus completely distinct from electromagnetic trapping.

In Section 2, a general expression for the potential energy of an optically trapped chiral molecule is first derived using quantum electrodynamics (QED) to elicit the specific photon-level detail of the underlying interaction. Section $\underline{3}$ then introduces the additional complications that arise because of the dynamic interaction of nonspherical chiral molecules with the trapping beam; this involves a weighted rotational average method to account for the orientational effects of the irradiating beam. The result provides for a quantitative estimate of the difference in dynamical behavior that arises for chiral molecules of opposite enantiomeric form, in a circularly polarized beam of Gaussian intensity profile. In particular, it is shown that a difference in the relative diffusion of each enantiomer, in and out of the most intense regions of the beam, affords a means of achieving enantiomer separation. The paper concludes in Section $\underline{4}$ with a discussion of some corollary practical issues.

\section{THEORY OF MOLECULES IN A TRAPPING BEAM}

\section{A. Time-Dependent Perturbation Theory}

To begin, we concisely summarize the background theory supporting the involvement of various multipoles in forward scattering. The most accurate theoretical framework to describe chiral discrimination in an optical trap is one in which the molecules and electromagnetic radiation are both quantized and treated on the same footing, i.e., the field vectors of semiclassical theory are promoted to operator status. This quantum electrodynamical procedure $[\underline{10}, \underline{11}]$ is used throughout the rest of this work, initially to derive a general expression for the potential energy of an optically trapped chiral molecule. The starting point to achieve the latter involves time-dependent perturbation theory [12], which, using the resolvent operator formalism, may be expressed as

$$
M_{F I} \equiv\langle F|M| I\rangle=\left\langle F\left|\sum_{p=0}^{\infty} H_{\mathrm{int}}\left(T_{0} H_{\mathrm{int}}\right)^{p}\right| I\right\rangle,
$$

where $M_{F I}$ is the matrix element, $|I\rangle$ and $|F\rangle$ denote the initial and final system states (incorporating both molecular and radiation states), $H_{\mathrm{int}}$ is the interaction Hamiltonian, $T_{0} \approx$ $\left(E_{I}-H_{0}\right)^{-1}$ in which $E_{I}$ is the energy of the initial state, and $H_{0}$ is the unperturbed Hamiltonian. For the phenomenon of electromagnetic trapping, the initial and final states are identical (namely, $M_{F I} \equiv M_{I I}$ ) and, therefore, only diagonal elements of the matrix element are significant, and Eq. (1) becomes an expectation value with respect to the operator $\bar{M}$. In such circumstances, there is no net transfer of energy, or linear or angular momentum from the radiation field to the molecule; trapping at the Mie-scale, where internal refraction leads to momentum transfer, is not considered in this work. The physical observable (potential energy, $U$ ) of electromagnetic trapping is simply found from the real part of $M_{I I}$ which, as a result Eq. (1), is rewritten as

$$
U=\operatorname{Re} M_{I I} \simeq \operatorname{Re}\left\{\sum_{R} \frac{\left\langle I\left|H_{\mathrm{int}}\right| R\right\rangle\left\langle R\left|H_{\mathrm{int}}\right| I\right\rangle}{E_{I}-E_{R}}\right\},
$$

where $R$ is an intermediate state, $E$ is the energy of the state denoted by its subscript, and $H_{\text {int }}$ is the interaction Hamiltonian that, in the multipolar formulation, is given by

$$
H_{\mathrm{int}}=-\varepsilon_{0}^{-1} \mu_{i} d_{i}^{\perp}-\varepsilon_{0}^{-1} Q_{i j} \nabla_{j} d_{i}^{\perp}-m_{i} b_{i}+\ldots,
$$

in which $\mu_{i}$ and $Q_{i j}$ are the electric dipole and quadrupole moment operators, respectively; $m_{i}$ is the magnetic dipole operator, and Einstein summation over repeated Cartesian indices $i, j$ is applied; the electric, $d_{i}^{\perp}$, and magnetic, $b_{i}$, operator components are expressed elsewhere [13]. The second and third terms on the right in Eq. (3) are of broadly similar magnitude, both deriving from the first spatial derivative of the vector potential, under canonical transformation.

\section{B. Potential Energy of an Electromagnetic Trap}

In most nanophotonic systems, the electric dipole $(E 1)$ interaction dominates over the magnetic dipole $(M 1)$ and electric quadrupole $(E 2)$ interactions, usually by two to three orders of magnitude. Typically, as stated earlier, the electric dipole approximation is used in optical trapping calculations, i.e., only the contributions of $E 1$ are considered, although the effects of $E 2$ interactions have been examined previously [14]. Chiral discriminatory studies, in contrast, require that the $M 1$ and $E 2$ interactions are also analyzed (both contributions emerge from the gradient of the electromagnetic vector potential, on canonical transformation to the multipolar form of interaction [15-17]). More precisely, taking the leading contributors only, the electromagnetic trapping potential energy comprises terms corresponding to electric dipole-electric dipole $(E 1-E 1)$, electric dipole-electric quadruple $(E 1-E 2)$, and electric dipole-magnetic dipole $(E 1-M 1)$ interactions, namely,

$$
U(\mathbf{r})=U_{E 1-E 1}(\mathbf{r})+U_{E 1-E 2}(\mathbf{r})+U_{E 1-M 1}(\mathbf{r}),
$$

the subscripts arising since Rayleigh scatting involves two photon-matter interactions. Assuming a circularly polarized plane wave input beam, and following insertion of the relevant term of Eqs. (3) into (2), each of the potential energy contributions in Eq. ( $\underline{4})$ emerges in a form expressible as follows [18]:

$$
\begin{gathered}
U_{E 1-E 1}^{(L \mid R)}(\mathbf{r})=\operatorname{Re}\left\{-\left(\frac{I(\mathbf{r})}{2 \varepsilon_{0} c}\right) \bar{e}_{i}^{(L \mid R)} e_{j}^{(L \mid R)} \alpha_{i j}\right\}, \\
U_{E 1-E 2}^{(L \mid R)}(\mathbf{r})=\operatorname{Re}\left\{-\left(\frac{i k_{k} I(\mathbf{r})}{2 \varepsilon_{0} c}\right) \bar{e}_{i}^{(L \mid R)} e_{j}^{(L \mid R)}\left(A_{i j k}-A_{j i k}\right)\right\}, \\
U_{E 1-M 1}^{(L \mid R)}(\mathbf{r})=\operatorname{Re}\left\{-\left(\frac{I(\mathbf{r})}{2 \varepsilon_{0} c^{2}}\right)\left(\bar{e}_{i}^{(L \mid R)} b_{j}^{(L \mid R)} G_{i j}\right.\right. \\
\left.\left.+\bar{b}_{i}^{(L \mid R)} e_{j}^{(L \mid R)} \bar{G}_{j i}\right)\right\} .
\end{gathered}
$$


Here, $I(\mathbf{r}) \equiv n(\mathbf{r}) \hbar c^{2} k / V$ signifies the irradiance of the input beam at a position $\mathbf{r}$, in which $\hbar c k$ is the energy of an input photon and $n$ is the number of photons within a quantization volume $V$; the latter is assumed to be positioned around the scattering molecule so that mean beam intensity at that position is $I(\mathbf{r})$. Moreover, $\mathbf{e}^{(L \mid R)}$ and $\mathbf{b}^{(L \mid R)}$ are the circular polarization of the electric and magnetic field, respectively, which is either left- or right-handed as denoted by the superscript; the over-bars represent complex conjugates, and $\mathbf{k}$ is the wave-vector of the input beam. Additionally, the electric dipolar-quadrupolar and electric-magnetic dipolar tensors (analogs to the polarizability tensor, $\alpha_{i j}$ ) are denoted by the symbols $A_{i j k}$ and $G_{i j}$. The explicit form of the latter two tensors, assuming that the energy of the input beam $(\hbar \omega)$ is within the transparent region of the molecule, are expressed as

$$
\begin{gathered}
A_{i j k}=\sum_{s}\left\{\frac{\mu_{i}^{0 s} Q_{j k}^{s 0}}{E_{s 0}-\hbar \omega}+\frac{Q_{j k}^{0 s} \mu_{i}^{s 0}}{E_{s 0}+\hbar \omega}\right\}, \\
G_{i j}=\sum_{s}\left\{\frac{\mu_{i}^{0 s} m_{j}^{s 0}}{E_{s 0}-\hbar \omega}+\frac{m_{j}^{0 s} \mu_{i}^{s 0}}{E_{s 0}+\hbar \omega}\right\} .
\end{gathered}
$$

where $\mu_{i}^{0 s}$ and $m_{i}^{0 s}$ are components of the transition electric and magnetic dipoles, for the transition $0 \leftarrow s$, respectively, and $Q_{j k}^{0 s}$ are components of the electric quadrupolar moment; $E_{s 0} \equiv E_{s}$ $E_{0}$ represents the energy difference between the molecular intermediate and ground state. The corresponding trapping force is found using the expression $\mathbf{F}^{(L \mid R)}(\mathbf{r})=-\nabla\left(U^{(L \mid R)}(\mathbf{r})\right)$, which gives a nonzero result, through $-\nabla I(\mathbf{r})$, unless the intensity of the irradiating beam or its derivative is null. An optical force of this type, and others, acting on chiral particles is currently of significant interest [19-22].

Upon examination of the dipolar-quadrupolar term, as given by Eq. (6), it is clear that the result must be null for cases when the input beam is distant from electronic resonance. This conclusion arises since nonresonance conditions signify that the result of Eq. (8) is real, meaning that the right-hand side of Eq. (6) is imaginary and, thus, inconsistent with potential energy (the observable) being a real quantity. As a result, only Eqs. (5) and (7) are to be considered in the following subsection, the term representative of electric quadrupole interactions having been proven to be unnecessary.

\section{Chiral Discrimination in an Electromagnetic Trap}

When a single enantiomer is irradiated with left-handed circularly polarized light, compared to right-handed light, a small variation in the measured observable may arise. This is known as chiral discrimination, and is the subject of much present interest, especially in connection with the detection [23-28] and optomechanical (or optofluidic) separation [29-40] of enantiomers.

For a chiral molecule within an electromagnetic trap, discrimination of this type is represented by the following expression:

$$
U^{(L-R)}(\mathbf{r})=i\left(\frac{I(\mathbf{r})}{2 \varepsilon_{0} c^{2}}\right)\left(\bar{e}_{i}^{(L)} e_{j}^{(L)}+\bar{e}_{i}^{(R)} e_{j}^{(R)}\right)\left(G_{i j}-\bar{G}_{j i}\right),
$$

as found from Eq. (4) using the identity $b_{i}^{(L \mid R)}=\bigotimes i e_{i}^{(L \mid R)}$ and where $U^{(L-R)} \equiv U^{(L)}-U^{(R)}$. Here, the $E 1-E 1$ contribution from Eq. (ㅁ) cancels out because of symmetry, i.e., an identical outcome is seen for left- and right-handed light, leaving the $E 1-M 1$ interaction as the sole contributor. A general result for chiral discrimination in an electromagnetic trap is found by simplifying Eq. (10), so that

$$
U^{(L-R)}(\mathbf{r})=-\left(\frac{I(\mathbf{r})}{\varepsilon_{0} c^{2}}\right)\left(\delta_{i j}-\hat{k}_{i} \hat{k}_{j}\right) G_{i j}^{\prime},
$$

with use of the identity $\bar{e}_{i}^{(L)} e_{j}^{(L)}+\bar{e}_{i}^{(R)} e_{j}^{(R)}=\delta_{i j}-\hat{k}_{i} \hat{k}_{j}$ and by setting $\operatorname{Im} G_{i j} \equiv G_{i j}^{\prime}$. Henceforth, for conciseness, any dependence on $\mathbf{r}$ will be suppressed. In Section 3, the latter expression will act as the starting point for an analysis on the orientational effects of the irradiating electromagnetic beam, and its influence on chiral discrimination.

\section{Enantiomer Separation in an Electromagnetic Trap}

Previously, for calculationally convenience, Eq. (11) was derived under the notion that the trapping force, $\mathbf{F}^{(L \mid R)}$, acting on a given enantiomer differs for left-handed polarized electromagnetic radiation compared with right-handed light. It is important to state, however, that identical expressions are determined when light of a single circular polarization is applied to a left-handed enantiomer relative to the right-handed one. Such a case leads to the interesting possibility of enantiomer separation, the corresponding chiral force being defined as $\Delta \mathbf{F} \equiv \mathbf{F}^{(L)}-\mathbf{F}^{(R)}$, where the superscripts are now representative of the enantiomer rather than the input radiation. This equivalence arises since right-handed light and a left-handed molecule, for instance, may be treated identically to left-handed light and a right-handed molecule.

A physical model for this prospect may involve a mixture of enantiomers of hexahelicene in a solution. Such molecules, which are emblematic of molecular chirality, are all attracted to the high intensity part of the trapping beam, for example, the center of a Gaussian beam. However, when the irradiating light is circularly polarized, the left-handed molecule, for example, may be more likely than its isomer to reside in this high intensity region (Fig. 1). Allowing for the effects of thermal motion in a solution, the chiral molecules will be constantly changing position within the beam with a differential chiral force $\Delta \mathbf{F}$, effectively pushing the $(L)$-hexahelicene toward the center (assuming Gaussian light is used) and repelling the $(R)$-hexahelicene. Non-chiral molecules would not be subjected to this force. To be clear, this prospect arises because of a totally independent, and subtly different, response of the leftand right-handed enantiomers to the trapping beam; intermolecular interactions, such as those relating to optical binding, are not entertained in this model. A further evaluation of our scheme is presented in the Discussion Section.

\section{ORIENTATIONAL EFFECTS OF THE INPUT BEAM}

A. Ensemble Averages in a Partially Oriented System

The previously determined results apply for a molecule with translational freedom but a fixed orientation. In these circumstances, upon extension to a many-particle system, the optical response of the single molecule is replicated 


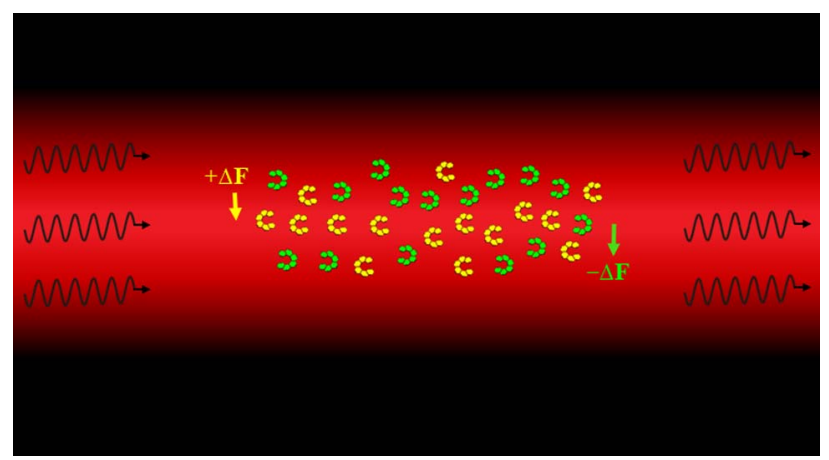

Fig. 1. Diagram depicting the relative positions, within a circularly polarized trapping beam of Gaussian profile, of two enantiomers: $(L)$ hexahelicene (yellow) and $(R)$-hexahelicene (green) in a solution. Both enantiomers are attracted to the center of the Gaussian beam, although, as denoted by the differential chiral force $\Delta \mathbf{F}$ (the optical force acting on the left-handed enantiomer compared with the right-handed one), a greater tendency arises for left-handed molecules to be positioned toward the beam center and right-handed enantiomers toward the periphery. Black wavy arrows denote the direction of the throughput light beam.

throughout the ensemble with respect to the angular disposition of the irradiating beam. However, direct correlations between the microcosm and macrocosm do not occur when the molecules hold a degree of orientational freedom. For partially oriented molecules within an ensemble, rather than those free to tumble, a suitable orientational distribution for the independent molecules is required with respect to the stimulus beam. Such an anisotropic case arises for the dynamic interactions of the input beam, i.e., through the tensor interaction $-\boldsymbol{\alpha} \cdot \boldsymbol{\varepsilon} \cdot \boldsymbol{\varepsilon}$ (where $\boldsymbol{\varepsilon}$ is the vector electric field of the laser), resulting in a partial orientation of the trapped molecules, compromised by thermal fluctuations. Modeled mathematically, this situation corresponds to an ensemble average involving a contraction between rank- 2 tensors for the field and for the molecule, additionally including weighting distributions that are also dependent on field-molecule rank-2 tensor contractions. Such an average, with the use of Eq. (11), is expressible in the following generic form:

$$
\left\langle U^{(L-R)}\right\rangle \equiv-\left(\frac{I}{\varepsilon_{0} c^{2}}\right) \frac{\langle\mathbf{A}: \mathbf{B} \exp (\mathbf{C}: \mathbf{D})\rangle}{\langle\exp (\mathbf{C}: \mathbf{D})\rangle},
$$

where $\mathbf{A}, \mathbf{B}, \mathbf{C}$, and $\mathbf{D}$ are all rank-2 tensors. The colons denote an inner product between two such tensors, and the chevrons signify an ensemble average [41]; $\mathbf{A}$ and $\mathbf{C}$ refer to the optical stimuli (in the laboratory frame); and $\mathbf{B}$ and $\mathbf{D}$ to the molecular response (molecule frame). This expression represents the dynamic analog to the much simpler physics associated with calculating a mean dipole in a static field, which includes a weighting distribution involving only rank-1 tensor (vector) contractions [42], and whose general form of weighting leads to the familiar Langevin function [43].

Explicitly, the rank-2 tensors of Eq. (12) are here defined as $\mathbf{A}=G_{i j}^{\prime}, \mathbf{B}=\delta_{i j}-\hat{k}_{i} \hat{k}_{j}, \mathbf{C}=\beta \alpha_{i j}\left(\beta=\varepsilon^{2} / k_{B} T\right.$, where the numerator is the square of an effective electric field and the denominator is a product of the Boltzmann constant and absolute temperature) and $\mathbf{D}=\frac{1}{2}\left(\delta_{i j}-\hat{k}_{i} \hat{k}_{j}\right)$, utilizing the weighting factor $-\boldsymbol{\alpha} \cdot \boldsymbol{\varepsilon} \cdot \boldsymbol{\varepsilon}$. Moreover, it is expedient for a decomposition of the reducible tensors to be introduced so that they are written as a sum of irreducible terms. For example, we can write $\mathbf{A}=\mathbf{A}^{(0)}+\mathbf{A}^{(1)}+\mathbf{A}^{(2)}$, where the superscripts denote the tensor weight: $\mathbf{A}^{(0)}$ transforms under rotations as a scalar, $\mathbf{A}^{(1)}$ as a pseudovector (even with respect to space inversion), and $\mathbf{A}^{(2)}$ as a symmetric, traceless second rank tensor [44]. Using these expression with Eq. (12), after a Taylor series expansion of the exponential function, the following is determined:

$$
\begin{aligned}
\left\langle U^{(L-R)}\right\rangle= & -\left(\frac{I}{\varepsilon_{0} c^{2}}\right)\left\{\left\langle\mathbf{A}^{(0)}: \mathbf{B}^{(0)}\right\rangle\right. \\
& \left.+\frac{\sum_{n=0}^{3} \frac{1}{n !}\left\langle\left(\mathbf{A}^{(2)}: \mathbf{B}^{(2)}\right)\left(\mathbf{C}^{(2)}: \mathbf{D}^{(2)}\right)^{n}\right\rangle}{\sum_{m=n+1}^{4} \frac{1}{m !}\left\langle\left(\mathbf{C}^{(2)}: \mathbf{D}^{(2)}\right)^{m}\right\rangle}\right\},
\end{aligned}
$$

where $\mathbf{A}^{(0)}=\frac{1}{3} \delta_{i j} G_{\lambda \lambda}^{\prime}, \mathbf{A}^{(2)}=\frac{1}{2}\left(G_{i j}^{\prime}+G_{j i}^{\prime}\right)-\frac{1}{3} \delta_{i j} G_{\lambda \lambda}^{\prime}, \mathbf{C}^{(0)}=$ $\frac{1}{3} \beta \delta_{i j} \alpha_{\lambda \lambda}, \mathbf{C}^{(2)}=\beta\left(\alpha_{i j}-\frac{1}{3} \delta_{i j} \alpha_{\lambda \lambda}\right), \mathbf{B}^{(0)}=2 \mathbf{D}^{(0)}=\frac{2}{3} \delta_{i j}, \mathbf{B}^{(2)}=$ $\frac{1}{3} \delta_{i j}-\hat{k}_{i} \hat{k}_{j}$, and $\mathbf{D}^{(2)}=\frac{1}{2}\left(\frac{1}{3} \delta_{i j}-\hat{k}_{i} \hat{k}_{j}\right)$. To continue, the relevant rank of rotational average $[45,46]$ now needs to be applied to each term of Eq. (13); thorough detail of this procedure can be found in Ref. [47]. The first term involves a simple second-rank rotational average, giving

$$
\left\langle\mathbf{A}^{(0)}: \mathbf{B}^{(0)}\right\rangle=2 G,
$$

in which $G=\frac{1}{3} G_{\lambda \lambda}^{\prime}$. On setting $n=0$, the second term also requires a second-rank average, which will equate to null, while $n=1$ requires a fourth-rank average entailing the use of a $3 \times 3$ matrix. The numerator of the second term is then determined as

$$
\left\langle\mathbf{A}^{(2)}: \mathbf{B}^{(2)}: \mathbf{C}^{(2)}: \mathbf{D}^{(2)}\right\rangle=\frac{\beta}{5}\left(G^{\alpha}-G \alpha\right),
$$

and the corresponding denominator is

$$
\frac{1}{2}\left\langle\mathbf{C}^{(2)}: \mathbf{D}^{(2)}: \mathbf{C}^{(2)}: \mathbf{D}^{(2)}\right\rangle=\frac{\beta}{10}\left(\alpha^{\alpha}-\alpha^{2}\right),
$$

employing $G^{\alpha}=\frac{1}{3} G_{\lambda \mu}^{\prime} \alpha_{\lambda \mu}, \alpha=\frac{1}{3} \alpha_{\lambda \lambda}$, and $\alpha^{\alpha}=\frac{1}{3} \alpha_{\lambda \mu} \alpha_{\lambda \mu}$. A more intricate sixth-rank rotational average, involving a $15 \times 15$-matrix, is needed when $n=2$. The numerator and denominator in such a case are

$$
\begin{gathered}
\frac{1}{2}\left\langle\mathbf{A}^{(2)}: \mathbf{B}^{(2)}: \mathbf{C}^{(2)}: \mathbf{D}^{(2)}: \mathbf{C}^{(2)}: \mathbf{D}^{(2)}\right\rangle \\
=-\frac{\beta^{2}}{35}\left(G^{\alpha \alpha}+G\left(2 \alpha^{2}-\alpha^{\alpha}\right)-2 G^{\alpha} \alpha\right), \\
\frac{1}{6}\left\langle\mathbf{C}^{(2)}: \mathbf{D}^{(2)}: \mathbf{C}^{(2)}: \mathbf{D}^{(2)}: \mathbf{C}^{(2)}: \mathbf{D}^{(2)}\right\rangle \\
=-\frac{\beta^{2}}{210}\left(\alpha^{\alpha \alpha}-3 \alpha^{\alpha} \alpha+2 \alpha^{3}\right),
\end{gathered}
$$

respectively, where the notations $G^{\alpha \alpha}=\frac{1}{6}\left(G_{\lambda \mu}^{\prime} \alpha_{\mu \nu} \alpha_{\nu \lambda}+\right.$ $\left.G_{\mu \lambda}^{\prime} \alpha_{\mu \nu} \alpha_{\nu \lambda}\right)$ and $\alpha^{\alpha \alpha}=\frac{1}{3} \alpha_{\lambda \mu} \alpha_{\mu \nu} \alpha_{\nu \lambda}$ are used. Upon setting $n=3$, a highly complicated eighth-rank rotational average [48] is necessary, a technique that has only very recently been 
applied to other physical systems $[\underline{49}, \underline{50}]$, so that the following expressions are found from explicit implementation of the relevant $105 \times 105$ matrix:

$$
\begin{aligned}
& \frac{1}{6}\left\langle\mathbf{A}^{(2)}: \mathbf{B}^{(2)}: \mathbf{C}^{(2)}: \mathbf{D}^{(2)}: \mathbf{C}^{(2)}: \mathbf{D}^{(2)}: \mathbf{C}^{(2)}: \mathbf{D}^{(2)}\right\rangle \\
& =\frac{\beta^{3}}{7250}\left(G^{\alpha}\left(39 \alpha^{\alpha}-9 \alpha^{2}\right)+9 G \alpha\left(\alpha^{2}-\alpha^{\alpha}\right)+10 G^{\alpha \alpha \alpha}\right. \\
& \left.\quad-10 G \alpha^{\alpha \alpha}\right), \\
& \frac{1}{24}\left\langle\mathbf{C}^{(2)}: \mathbf{D}^{(2)}: \mathbf{C}^{(2)}: \mathbf{D}^{(2)}: \mathbf{C}^{(2)}: \mathbf{D}^{(2)}: \mathbf{C}^{(2)}: \mathbf{D}^{(2)}\right\rangle \\
& =\frac{\beta^{3}}{58000}\left(3 \alpha^{\alpha}\left(13 \alpha^{\alpha}-6 \alpha^{2}\right)+10 \alpha^{\alpha \alpha \alpha}+40 \alpha^{\alpha \alpha} \alpha+9 \alpha^{4}\right)
\end{aligned}
$$

Here, $\alpha^{\alpha \alpha \alpha}=\frac{1}{3} \alpha_{\lambda \mu} \alpha_{\mu \nu} \alpha_{\nu o} \alpha_{o \lambda}$ and $G^{\alpha \alpha \alpha}=\frac{1}{6}\left(G_{\lambda \mu}^{\prime} \alpha_{\mu \nu} \alpha_{\nu o} \alpha_{o \lambda}+\right.$ $\left.G_{\mu \lambda}^{\prime} \alpha_{\mu \nu} \alpha_{\nu o} \alpha_{o \lambda}\right)$. By inserting Eqs. (14)-(20) into Eq. (13), the following expression is revealed:

$$
\begin{aligned}
\left\langle U^{(L-R)}\right\rangle & =-\left(\frac{I}{\varepsilon_{0} c^{2}}\right)\left\{2 G+2 \beta\left[G^{\alpha}-G \alpha-\frac{\beta}{21}\left(G^{\alpha \alpha}+3 G\right.\right.\right. \\
& \left.\times\left(2 \alpha^{2}-\alpha^{\alpha}\right)-6 G^{\alpha} \alpha\right)+\frac{\beta^{2}}{1450}\left(3 G^{\alpha}\left(13 \alpha^{\alpha}-3 \alpha^{2}\right)+9 G \alpha\right. \\
& \left.\left.\times\left(\alpha^{2}-\alpha^{\alpha}\right)+10\left(G^{\alpha \alpha \alpha}-3 G^{\alpha \alpha} \alpha-2 G \alpha^{\alpha \alpha}\right)\right)\right] \\
& \times\left(10+\beta^{2}\left[\alpha^{\alpha}-\alpha^{2}-\frac{\beta}{21}\left(\alpha^{\alpha \alpha}+\alpha\left(2 \alpha^{2}-3 \alpha^{\alpha}\right)\right)\right.\right. \\
& +\frac{\beta^{2}}{58000}\left(3 \alpha^{\alpha}\left(13 \alpha^{\alpha}-6 \alpha^{2}\right)\right. \\
& \left.\left.\left.\left.+10 \alpha^{\alpha \alpha \alpha}-40 \alpha^{\alpha \alpha} \alpha+9 \alpha^{4}\right)\right]\right)^{-1}\right\}
\end{aligned}
$$

This expression shows the difference in potential energy that arises on comparison of enantiomers with opposite handiness in an electromagnetic trap, accounting for the partially orienting effects of the input beam.

If, to further simplify Eq. (21) to a form involving fewer parameters, it is further assumed that the chiral molecules have essentially isotropic $\alpha$ and $G$ tensors [51-56], the following is obtained:

$$
\begin{aligned}
\left\langle U^{(L-R)}\right\rangle= & -\left(\frac{I g}{\varepsilon_{0} c^{2}}\right)\left\{1+\beta\left(2 a+\frac{4}{7} \beta a^{2}+\frac{63}{725} \beta^{2} a^{3}\right)\right. \\
& \left.\times\left(5+\beta^{2}\left(a^{2}-\frac{1}{21} \beta a^{3}+\frac{27}{14500} \beta^{2} a^{4}\right)\right)^{-1}\right\},
\end{aligned}
$$

where $a=\alpha$ and $g=G$ are the scalar representatives of isotropic electric-electric and electric-magnetic scattering tensors, respectively, and the previous symbols are redefined as $G^{\alpha}=3 a g, \quad \alpha^{\alpha}=3 a^{2}, \quad G^{\alpha \alpha}=9 a^{2} g, \quad \alpha^{\alpha \alpha}=9 a^{3}, \quad G^{\alpha \alpha \alpha}=$ $27 a^{3} g$, and $\alpha^{\alpha \alpha \alpha}=27 a^{4}$.

\section{B. Limiting Cases for the Electromagnetic Trap}

The isotropic result, where the molecules are allowed to freely tumble, may easily be recovered from Eq. (21). This is achieved by setting $\beta \rightarrow 0$, physically denoting an input beam with a very small electric field (or a system with very high temperatures), so that Eq. (21) is rewritten as $\left\langle U^{(L-R)}\right\rangle=-2 G I / \varepsilon_{0} c^{2}$. Alternatively, the result may be derived by the insertion of Eq. (14) into the first (isotropic) term of Eq. (13). At the other extreme, where the radiant electric field $\varepsilon$ is very large, or the temperatures are very low, the subsequent expression is resolved from Eq. (21):

$$
\begin{aligned}
\left\langle U^{(L-R)}\right\rangle= & -\left(\frac{80 I}{\varepsilon_{0} c^{2}}\right)\left\{3 G^{\alpha}\left(13 \alpha^{\alpha}-3 \alpha^{2}\right)+9 G \alpha\left(\alpha^{2}-\alpha^{\alpha}\right)\right. \\
& \left.+10\left(G^{\alpha \alpha \alpha}-3 G^{\alpha \alpha} \alpha-2 G \alpha^{\alpha \alpha}\right)\right\}\left(3 \alpha^{\alpha}\left(13 \alpha^{\alpha}-6 \alpha^{2}\right)\right. \\
& \left.+10 \alpha^{\alpha \alpha \alpha}-40 \alpha^{\alpha \alpha} \alpha+9 \alpha^{4}\right)^{-1},
\end{aligned}
$$

which is found by taking the terms associated with the highest order of $\beta$ in the numerator and denominator. Supposing essentially isotropic $\alpha$ and $G$ tensors, Eq. (23) becomes

$$
\left\langle U^{(L-R)}\right\rangle=-\left(\frac{140 I}{3 \varepsilon_{0} c^{2}}\right)\left(\frac{g}{\beta a}\right),
$$

which can also be found directly from Eq. (22).

\section{DISCUSSION}

Using Eq. (24), the differential $E 1-M 1$ effect is determined to be roughly $1 / 6$ th that of the $E 1-E 1$ contribution. The total force for one of the enantiomer is the addition of the $E 1-E 1$ and $E 1-M 1$ couplings, while the other enantiomer involves subtracting the $E 1-M 1$ from the $E 1-E 1$ term. Therefore, the difference (i.e., the chiral force) between the two enantiomers is $F_{0} / 6$, where $F_{0}$ is the optical trapping force resulting from the $E 1-E 1$ interaction. This is the origin of the $\Delta \mathbf{F}$ discrimination forces shown in Fig. 1 .

More detailed calculations, based on a specific evaluation of the differential forces using $\mathbf{F}^{(L \mid R)}(\mathbf{r})=-\nabla\left(U^{(L \mid R)}(\mathbf{r})\right)$, show, for example, that at room temperature, even a comparatively small molecule with a polarizability volume $\left(\alpha / 4 \pi \varepsilon_{0}\right)$ of $10^{-29} \mathrm{~m}^{3}$, in a beam of intensity $5 \times 10^{11} \mathrm{Wcm}^{-2}$ and beam waist $10 \mu \mathrm{m}$, will experience differential forces of $10^{-16} \mathrm{~N}$ or more-a figure considered well within experimental reach [35]. Comparing this value to the thermodynamic force associated with molecular diffusion, against a concentration gradient (from Fick's first law) [57], it emerges that a force on this scale should be sufficient to sustain a gradient of $\sim 0.5 \%$ in the differential concentrations of enantiomers with opposite handedness, $(\Delta C / C)$, per cycle. Consequently, in a beam with a typically Gaussian profile, the result will be a greater tendency for molecules of one handedness to be positioned nearer the beam center, with molecules of the opposite handedness more often near the periphery. Larger molecules such as those proposed in our earlier scheme, i.e., hexahelicene, can be anticipated to deliver forces that are larger by at least an order of magnitude, with correspondingly larger concentration gradients.

Moving beyond the initial assumption of plane wave (paraxial) light, it is evident that other contributions may arise under the conditions of tightly focused beams [14] or light with complex waveforms [58], which may further enhance the optical forces - thus delivering scope for future investigations. 
In conclusion, it appears entirely realistic to devise new means of differentiating and separating mixtures of chiral enantiomers in a solution, based on their differential response to circularly polarized light.

It is our hope that this work, inspired by the International Year of Light and Light-Based Technologies, represents a modest contribution to the ongoing progress in molecular optics. The authors gratefully acknowledge funding from the Leverhulme Trust.

\section{REFERENCES}

1. G. Cipparrone, A. Mazzulla, A. Pane, R. J. Hernandez, and R. Bartolino, "Chiral self-assembled solid microspheres: a novel multifunctional microphotonic device," Adv. Mater. 23, 5773-5778 (2011).

2. R. J. Hernandez, A. Mazzulla, A. Pane, K. Volke-Sepulveda, and G. Cipparrone, "Attractive-repulsive dynamics on light-responsive chiral microparticles induced by polarized tweezers," Lab Chip 13, 459-467 (2013).

3. M. G. Donato, J. Hernandez, A. Mazzulla, C. Provenzano, R. Saija, R. Sayed, S. Vasi, A. Magazzù, P. Pagliusi, R. Bartolino, P. G. Gucciardi, O. M. Maragò, and G. Cipparrone, "Polarization-dependent optomechanics mediated by chiral microresonators," Nat. Commun. 5, 3656 (2014).

4. G. Tkachenko and E. Brasselet, "Helicity-dependent threedimensional optical trapping of chiral microparticles," Nat. Commun. 5, 4491 (2014).

5. M. Dienerowitz, M. Mazilu, and K. Dholakia, "Optical manipulation of nanoparticles: a review," J. Nanophoton. 2, 021875 (2008).

6. T. Čižmár, L. C. Dávila Romero, K. Dholakia, and D. L. Andrews, "Multiple optical trapping and binding: new routes to self-assembly," J. Phys. B 43, 102001 (2010).

7. O. M. Marago, P. H. Jones, P. G. Gucciardi, G. Volpe, and A. C. Ferrari, "Optical trapping and manipulation of nanostructures," Nat. Nanotechnol. 8, 807-819 (2013).

8. R. W. Bowman and M. J. Padgett, "Optical trapping and binding," Rep. Prog. Phys. 76, 026401 (2013)

9. D. S. Bradshaw and D. L. Andrews, "Interparticle interactions: energy potentials, energy transfer, and nanoscale mechanical motion in response to optical radiation," J. Phys. Chem. A 117, 75-82 (2013).

10. D. P. Craig and T. Thirunamachandran, Molecular Quantum Electrodynamics: An Introduction to Radiation-Molecule Interactions (Dover, 1998).

11. A. Salam, Molecular Quantum Electrodynamics: Long-Range Intermolecular Interactions (Wiley, 2009).

12. C. Cohen-Tannoudji, J. Dupont-Roc, and G. Grynberg, Atom-Photon Interactions: Basic Processes and Applications (Wiley, 1992).

13. D. L. Andrews and D. S. Bradshaw, "Virtual photons, dipole fields and energy transfer: a quantum electrodynamical approach," Eur. J. Phys. 25, 845-858 (2004).

14. V. V. Klimov and V. S. Letokhov, "Gradient optical force on atoms: beyond dipole approximation," Opt. Commun. 126, 45-48 (1996).

15. E. A. Power and T. Thirunamachandran, "On the nature of Hamiltonian for interaction of radiation with atoms and molecules: (e/mc)p.A, - $\mu . E$, and all that," Am. J. Phys. 46, 370-378 (1978).

16. E. A. Power and T. Thirunamachandran, "The multipolar Hamiltonian in radiation theory," Proc. R. Soc. A 372, 265-273 (1980).

17. R. G. Woolley, "Gauge invariance in non-relativistic electrodynamics," Proc. R. Soc. A 456, 1803-1819 (2000).

18. D. S. Bradshaw and D. L. Andrews, "Chiral discrimination in optical trapping and manipulation," New J. Phys. 16, 103021 (2014).

19. D. T. Butcher, S. Y. Buhmann, and S. Scheel, "Casimir-Polder forces between chiral objects," New J. Phys. 14, 113013 (2012).

20. Q.-C. Shang, Z.-S. Wu, T. Qu, Z.-J. Li, L. Bai, and L. Gong, "Analysis of the radiation force and torque exerted on a chiral sphere by a Gaussian beam," Opt. Express 21, 8677-8688 (2013).
21. K. Ding, J. Ng, L. Zhou, and C. T. Chan, "Realization of optical pulling forces using chirality," Phys. Rev. A 89, 063825 (2014).

22. S. B. Wang and C. T. Chan, "Lateral optical force on chiral particles near a surface," Nat. Commun. 5, 3307 (2014).

23. D. Patterson, M. Schnell, and J. M. Doyle, "Enantiomer-specific detection of chiral molecules via microwave spectroscopy," Nature 497 475-477 (2013).

24. D. Patterson and J. M. Doyle, "Sensitive chiral analysis via microwave three-wave mixing," Phys. Rev. Lett. 111, 023008 (2013).

25. M. H. M. Janssen and I. Powis, "Detecting chirality in molecules by imaging photoelectron circular dichroism," Phys. Chem. Chem. Phys. 16, 856-871 (2014).

26. D. Patterson and M. Schnell, "New studies on molecular chirality in the gas phase: enantiomer differentiation and determination of enantiomeric excess," Phys. Chem. Chem. Phys. 16, 11114-11123 (2014).

27. M. Okuno and T.-A. Ishibashi, "Chirality discriminated by heterodynedetected vibrational sum frequency generation," J. Phys. Chem. Lett. 5, 2874-2878 (2014).

28. V. A. Shubert, D. Schmitz, D. Patterson, J. M. Doyle, and M. Schnell, "Identifying enantiomers in mixtures of chiral molecules with broadband microwave spectroscopy," Angew. Chem. Int. Ed. 53, 11521155 (2014)

29. P. Král, I. Thanopulos, M. Shapiro, and D. Cohen, "Two-step enantioselective optical switch," Phys. Rev. Lett. 90, 033001 (2003).

30. Y. Li, C. Bruder, and C. P. Sun, "Generalized Stern-Gerlach effect for chiral molecules," Phys. Rev. Lett. 99, 130403 (2007).

31. W. Z. Jia and L. F. Wei, "Distinguishing left- and right-handed molecules using two-step coherent pulses," J. Phys. B 43, 185402 (2010).

32. A. Eilam and M. Shapiro, "Spatial separation of dimers of chiral molecules," Phys. Rev. Lett. 110, 213004 (2013).

33. X. Li and M. Shapiro, "Spatial separation of enantiomers by coherent optical means," J. Chem. Phys. 132, 041101 (2010).

34. X. Li and M. Shapiro, "Theory of the optical spatial separation of racemic mixtures of chiral molecules," J. Chem. Phys. 132, 194315 (2010).

35. A. Canaguier-Durand, J. A. Hutchison, C. Genet, and T. W. Ebbesen, "Mechanical separation of chiral dipoles by chiral light," New J. Phys. 15, 123037 (2013).

36. R. P. Cameron, S. M. Barnett, and A. M. Yao, "Discriminatory optical force for chiral molecules," New J. Phys. 16, 013020 (2014).

37. R. P. Cameron, A. M. Yao, and S. M. Barnett, "Diffraction gratings for chiral molecules and their applications," J. Phys. Chem. A 118, 34723478 (2014)

38. R. P. Cameron and S. Barnett, "Optical activity in the scattering of structured light," Phys. Chem. Chem. Phys. 16, 25819-25829 (2014).

39. A. Canaguier-Durand and C. Genet, "Chiral near fields generated from plasmonic optical lattices," Phys. Rev. A 90, 023842 (2014).

40. G. Tkachenko and E. Brasselet, "Optofluidic sorting of material chirality by chiral light," Nat. Commun. 5, 3577 (2014).

41. S. N. A. Smith and D. L. Andrews, "Three-dimensional ensemble averages for tensorial interactions in partially oriented, multi-particle systems," J. Phys. A 44, 395001 (2011).

42. D. L. Andrews and M. J. Harlow, "Phased and Boltzmann-weighted rotational averages," Phys. Rev. A 29, 2796-2806 (1984).

43. C. E. Hecht, Statistical Thermodynamics and Kinetic Theory (W. H. Freeman, 1990)

44. D. L. Andrews and P. Allcock, Optical Harmonics in Molecular Systems (Wiley-VCH, 2002).

45. D. L. Andrews and T. Thirunamachandran, "On threedimensional rotational averages," J. Chem. Phys. 67, 5026-5033 (1977).

46. D. H. Friese, M. T. P. Beerepoot, and K. Ruud, "Rotational averaging of multiphoton absorption cross sections," J. Chem. Phys. 141 204103 (2014).

47. J. S. Ford and D. L. Andrews, "One- and two-photon absorption in solution: the effects of a passive auxiliary beam," J. Chem. Phys. 141, 034504 (2014) 
48. D. L. Andrews and W. A. Ghoul, "Eighth-rank isotropic tensors and rotational averages," J. Phys. A 14, 1281-1290 (1981).

49. J. M. Leeder, D. S. Bradshaw, and D. L. Andrews, "Lasercontrolled fluorescence in two-level systems," J. Phys. Chem. B 115, 5227-5233 (2011).

50. J. M. Leeder and D. L. Andrews, "A molecular theory for two-photon and three-photon fluorescence polarization," J. Chem. Phys. 134, 094503 (2011).

51. D. V. Guzatov and V. V. Klimov, "Chiral particles in a circularly polarised light field: new effects and applications," Quantum Electron. 41, 526-533 (2011).

52. V. V. Klimov, D. V. Guzatov, and M. Ducloy, "Engineering of radiation of optically active molecules with chiral nano-metaparticles," Europhys. Lett. 97, 47004 (2012).
53. D. V. Guzatov and V. V. Klimov, "The influence of chiral spherical particles on the radiation of optically active molecules," New J. Phys. 14, 123009 (2012).

54. Z.-S. Wu, Q.-C. Shang, and Z.-J. Li, "Calculation of electromagnetic scattering by a large chiral sphere," Appl. Opt. 51, 6661-6668 (2012).

55. Q.-C. Shang, Z.-S. Wu, T. Qu, Z.-J. Li, L. Bai, and L. Gong, "Analysis of rainbow scattering by a chiral sphere," Opt. Express 21, 2187921888 (2013).

56. V. V. Klimov, I. V. Zabkov, A. A. Pavlov, and D. V. Guzatov, "Eigen oscillations of a chiral sphere and their influence on radiation of chiral molecules," Opt. Express 22, 18564-18578 (2014).

57. P. W. Atkins and J. De Paula, Atkins' Physical Chemistry (Oxford University, 2010).

58. D. L. Andrews and M. Babiker, The Angular Momentum of Light (Cambridge University, 2013). 